\title{
The utility of KRAS mutation analysis in differentiating a lung primary mucinous carcinoma from a metastatic colorectal mucinous carcinoma
}

\author{
Mahmoud L. Soliman, Shi Yang, John Cho Lee \\ Department of Pathology and Laboratory Medicine, Boston University Medical Center, Boston, Massachusetts, USA
}

Received: November 16, 2017

DOI: $10.5430 /$ crcp.v4n4p17
Accepted: December 7, 2017

Online Published: December 13, 2017

\begin{abstract}
A 58-year-old male has a past medical history of a rectal low-grade mucinous adenocarcinoma with a KRAS codon 12/13 mutation (GGT>GAT), for which he received neoadjuvant chemotherapy and radiation. Five months after diagnosis, the patient underwent a low anterior resection showing persistent tumor with the pathological staging being (ypT3, ypN0, ypMx). Six months after surgery, follow-up PET scan showed two right upper lung nodules measuring 1.0 and $1.8 \mathrm{~cm}$. Subsequent video-assisted thoracic surgery with a lung wedge resection revealed two tumors: a mucinous adenocarcinoma and an acinar-predominant adenocarcinoma. The mucinous adenocarcinoma showed similar cytologic features as the rectal tumor; however, it showed more of a lepidic pattern. The immunohictochemical profile of the lung mucinous adenocarcinoma was positive CK7 (cytoplasmic), negative CK20, TTF1, napsin-A, and CDX2. KRAS codon 12/13 analysis also showed a mutation in the lung, however it was different compared to the previous rectal tumor and showed a GGT>AGT mutation. The acinar adenocarcinoma had a wild-type KRAS. In this case, we favored a primary lung mucinous carcinoma over a metastasis from the rectum based on the lepidic morphology, CK20 negativity and differing KRAS codon 12 mutations. A year after the resection of the lung adenocarcinomas, the patient presented with dyspnea and abnormal liver functions. Abdominal US and MRI revealed multiple liver lesions (up to $1.9 \mathrm{~cm}$ ). Core liver biopsies showed a poorly-differentiated mucinous adenocarcinoma with positive CK7 (cytoplasmic) and negative CK20, TTF-1, napsin-A, CDX2 and HSA. KRAS codon 12/13 analysis also showed a mutation GGT>AGT, similar to that of the lung mucinous adenocarcinoma. The immunohistochemical profile and the KRAS mutation sequence of the hepatic tumor suggests a metastasis from the lung primary and corroborates the earlier premise that the lung tumor is distinct from the rectal one. In conclusion, in the unusual circumstance of a lung mucinous adenocarcinoma in a patient with established gastrointestinal mucinous primary, KRAS mutation analysis sequencing could help distinguish whether the lung mucinous is a primary tumor or metastasis from colorectal origin.
\end{abstract}

Key Words: KRAS, Mucinous, Adenocarcinoma, Lung, Colorectal

\section{CASe Presention}

A 58-year old male with a history of chronic obstructive pulmonary disease, smoking, hepatitis $\mathrm{C}$, cirrhosis, hemorrhoids, obesity, and cervical radiculopathy presented with a chief complaint of bright red blood per rectum. Colonoscopy revealed an ulcerated, non-obstructing, partially circumferential mass involving $40 \%$ of the recto-sigmoid colon, as well as an $8 \mathrm{~mm}$ flat polyp in the mid ascending colon and

\footnotetext{
* Correspondence: Mahmoud L. Soliman; Email: mahmoud.soliman@bmc.org; Address: Department of Pathology and Laboratory Medicine Boston University Medical Center 670 Albany St, suite 675 Boston, MA 02118, USA.
} 
multiple diverticula. Histopathological examination of the ulcerated mass revealed an invasive moderately-differentiated mucinous adenocarcinoma with the polyp being a tubular adenoma with high-grade dysplasia. Molecular analysis revealed that this carcinoma carries a KRAS mutation: c.35G $>$ A (p.G12D, codon 12 GGT $>$ GAT) and wild-type BRAF. Immunohistochemical studies showed intact expression of MLH1, MSH2, PMS2, and MSH6 indicating mismatch repair proficiency and negative screening for Lynch syndrome. The patient underwent neoadjuvant chemotherapy for one month with capecitabine concomitant with one month of daily radiation (prescribed dose of 5,000 cGy, prescribed fraction of 25 and prescribed dose/fraction of 200). A followup pelvic MRI showed a residual mass occluding $75 \%$ of the rectum located $10-11 \mathrm{~cm}$ from the anal verge. The patient underwent low anterior resection with diverting ileostomy four months later. On gross pathologic examination, the tumor appeared as an exophytic irregular mass measuring $4.5 \mathrm{~cm} \times$ $3.0 \mathrm{~cm} \times 0.8 \mathrm{~cm}$ that is located below the peritoneal reflection (see Figure 1A). Histologically, the tumor appeared to be a low-grade (well to moderately-differentiated) mucinous adenocarcinoma invading through the muscularis propria into the perirectal adipose tissue (see Figures 1B \& C). Minimal treatment effect was noted. No perineural nor lymphvascular invasion was seen. No metastasis was detected in twenty lymph nodes and no discontinuous extramural extension was seen. All margins were uninvolved by tumor. The tumor was assigned an American Joint of Committee on Cancer (AJCC) 7th edition pathologic stage yIIA (ypT3, ypN0). After surgery, adjuvant chemotherapy involving eight cycles of FOLFOX regimen was administered.

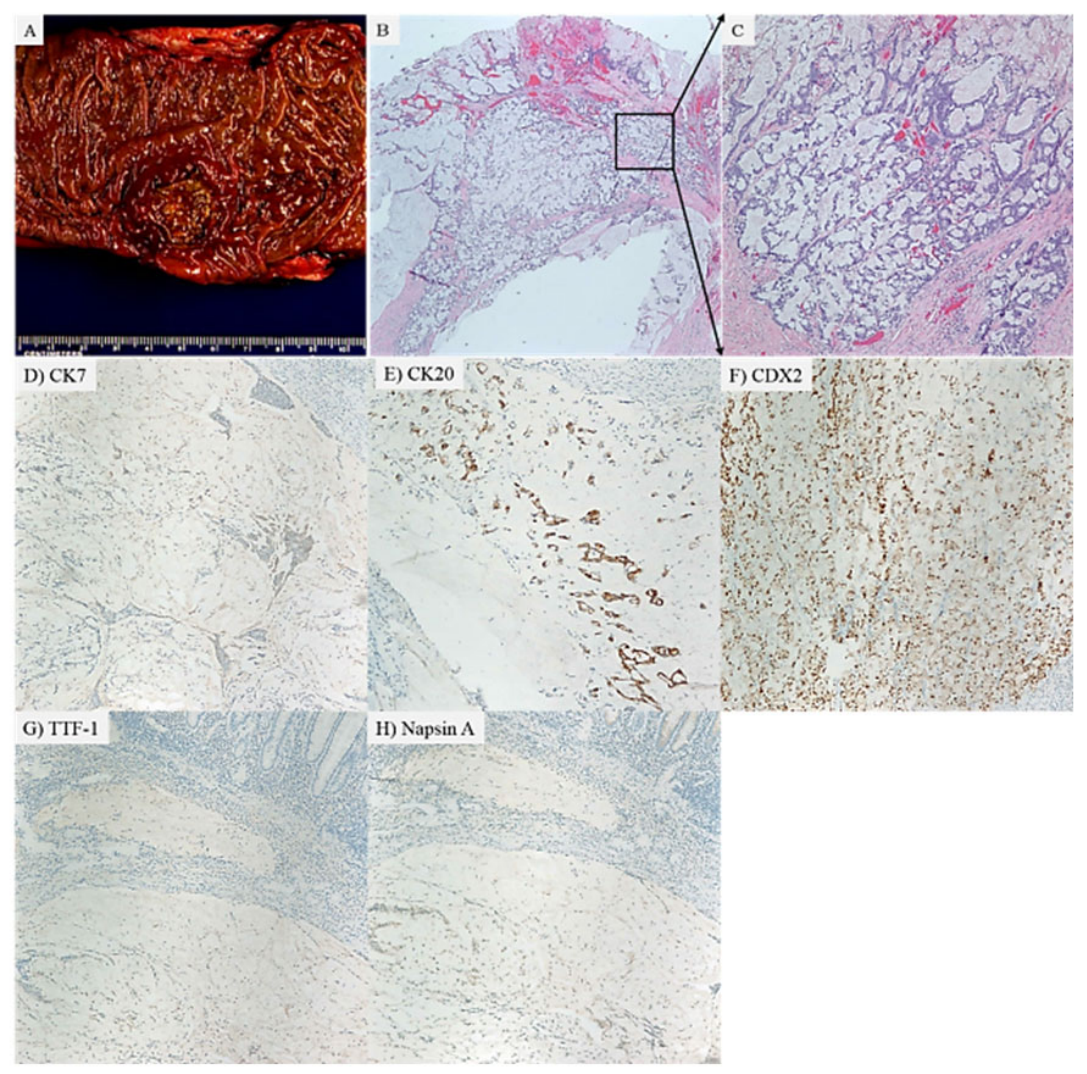

Figure 1. A) Gross picture of the rectal mass, B \& C) H\&E stain at 40x and 100x respectively, showing mucinous adenocarcinoma. D) through $\mathrm{H}$ ) 100x, the immunohistochemical profile of the rectal mucinous adenocarcinoma shows negative staining for CK7, positive cytoplasmic CK20 staining, diffuse and strong nuclear staining for CDX2, and negative staining for TTF-1 and napsin-A.

After the completion of the adjuvant chemotherapy, a restaging $\mathrm{CT}$ and PET scans showed an enlarging right upper lobe lung nodule measuring $1.0 \mathrm{~cm} \times 1.0 \mathrm{~cm}$ concerning for metastasis. Video-assisted thoracoscopic surgery-mediated right upper lobe wedge resection was performed. Histopathological examination revealed two different adenocarcinomas measuring $1.6 \mathrm{~cm} \times 0.4 \mathrm{~cm}$ and $1.3 \mathrm{~cm} \times 0.8 \mathrm{~cm}$ which were $3.0 \mathrm{~cm}$ apart from one another. The larger adenocarcinoma 
showed acinar histology (see Figure 2A) with the following immunohistochemical profile: CK7+, CK20-/+, TTF-1-/+, Napsin A+/- and CDX2- (see Figures 2B through F). CK7 diffusely positive (cytoplasmic), CK20 minor subset faintly positive (cytoplasmic), CDX2 negative, TTF-1 minor subset positive (nuclear), and napsin-A mostly strongly positive (cytoplasmic). The smaller adenocarcinoma showed mucinous histology (see Figure 3A) with a lepidic pattern and the following immunohistochemical profile: CK7+, CK20-, TTF-1/+, Napsin A-/+, CDX2-/+ (see Figures 3B through F). CK7 diffusely and strongly positive (cytoplasmic), CK20 negative, CDX2 negative, TTF-1 mostly negative with a minor subset positive (nuclear), and napsin-A mostly negative. No visceral pleural or lymphvascular invasion was noted. The resection margin was uninvolved by tumor. Molecular analysis revealed a different KRAS codon 12 mutation: c.34G $>$ A (p.G12S, codon 12 GGT>AGT) in the mucinous lung adenocarcinoma, with the acinar-predominant adenocarcinoma being KRAS wildtype. The AJCC 7th Edition Pathologic Staging for the lung cancers was IA (pT1a, pN0, pMX). This clinical scenario posed the question as to whether the mucinous adenocarcinoma in the lung is a metastasis from the rectum or an unrelated lung primary. Of note, surgical resection was followed by 45 days of chest radiation (prescribed dose of 4,600 cGy, prescribed fraction of 23 and prescribed dose/fraction of $200 \mathrm{cGy}$ ).

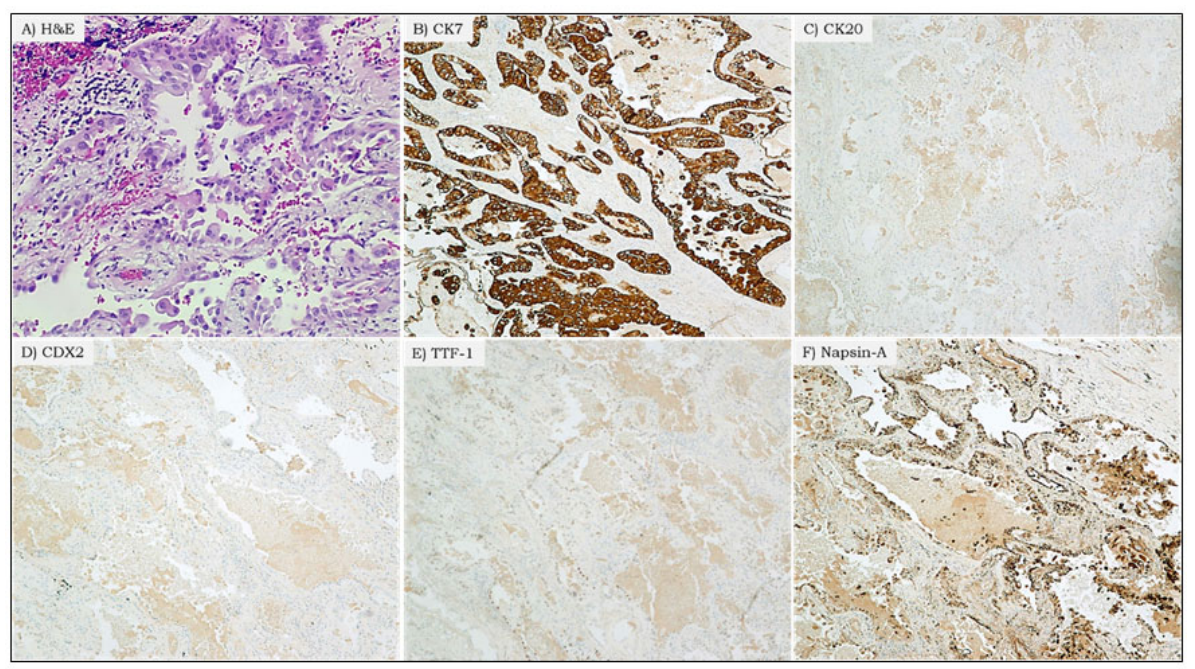

Figure 2. A) H\&E at 100x showing adenocarcinoma of the lung (acinar pattern). B) through F) The immunohistochemical profile: CK7 diffusely positive (cytoplasmic), CK20 minor subset faintly positive (cytoplasmic), CDX2 negative, TTF-1 minor subset positive (nuclear), and napsin-A mostly strongly positive (cytoplasmic)

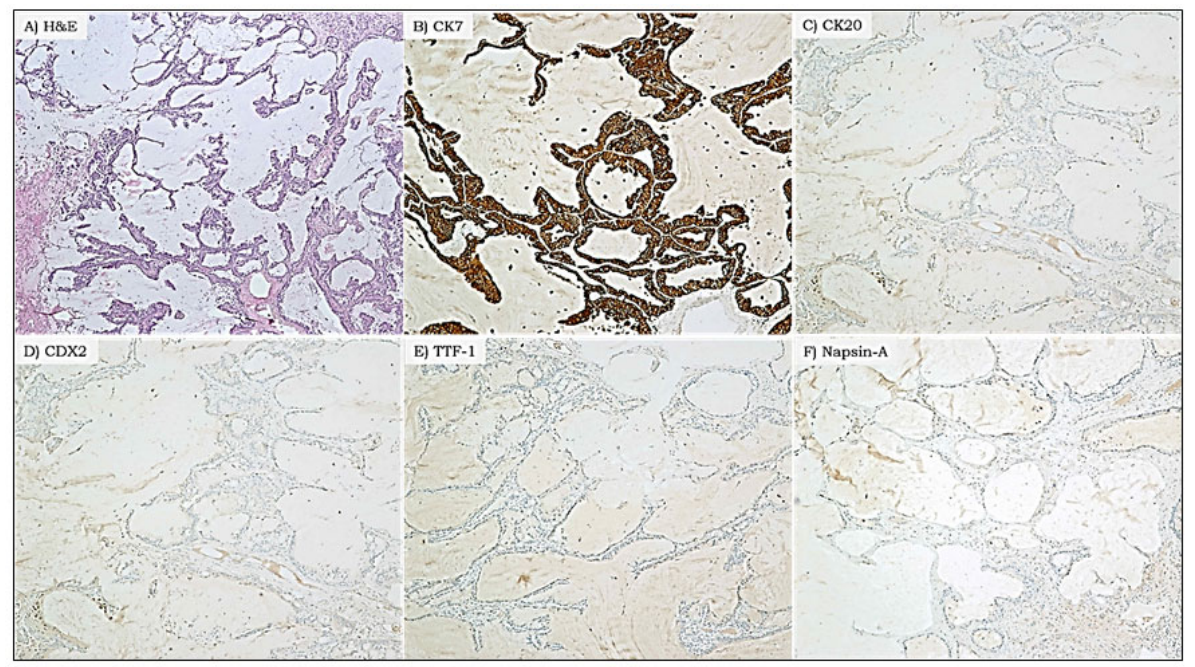

Figure 3. A) H\&E stain 100x showing adenocarcinoma of the lung (mucinous). B) through F) The immunohistochemical profile: CK7 diffusely and strongly positive (cytoplasmic), CK20 negative, CDX2 negative, TTF-1 mostly negative with a minor subset positive (nuclear), and napsin-A mostly negative. 
A year later the patient presented with a complaint of shortness of breath. Hepatic and biliary laboratory abnormalities and the history of rectal and lung tumors insitigated abdominal US and subsequent MRI revealing multiple liver metastases with the largest in segment VIII measuring $1.9 \mathrm{~cm}$ $\times 1.9 \mathrm{~cm}$. Histopathological examination of two liver core biopsies showed a poorly-differentiated adenocarcinoma (see Figure 4A) with the following immunohistochemical profile: CK7+, CK20-, CDX-2-/+, TTF-1-/+, Napsin A-/+ and HAS- (see Figures 4B through $\mathrm{G}$ ) compatible with metastasis from a lung primary. CK7 strongly and diffusely positive (cytoplasmic), CK20 negative, CDX2 negative, TTF-1 negative, napsin A negative, and HAS negative. Chemotherapy with carboplatin/pemetrexed was initiated but had to be stopped and switched to nivolumab due to thrombocytopenia. The patient died shortly after from complications of acute liver failure and lactic acidosis.

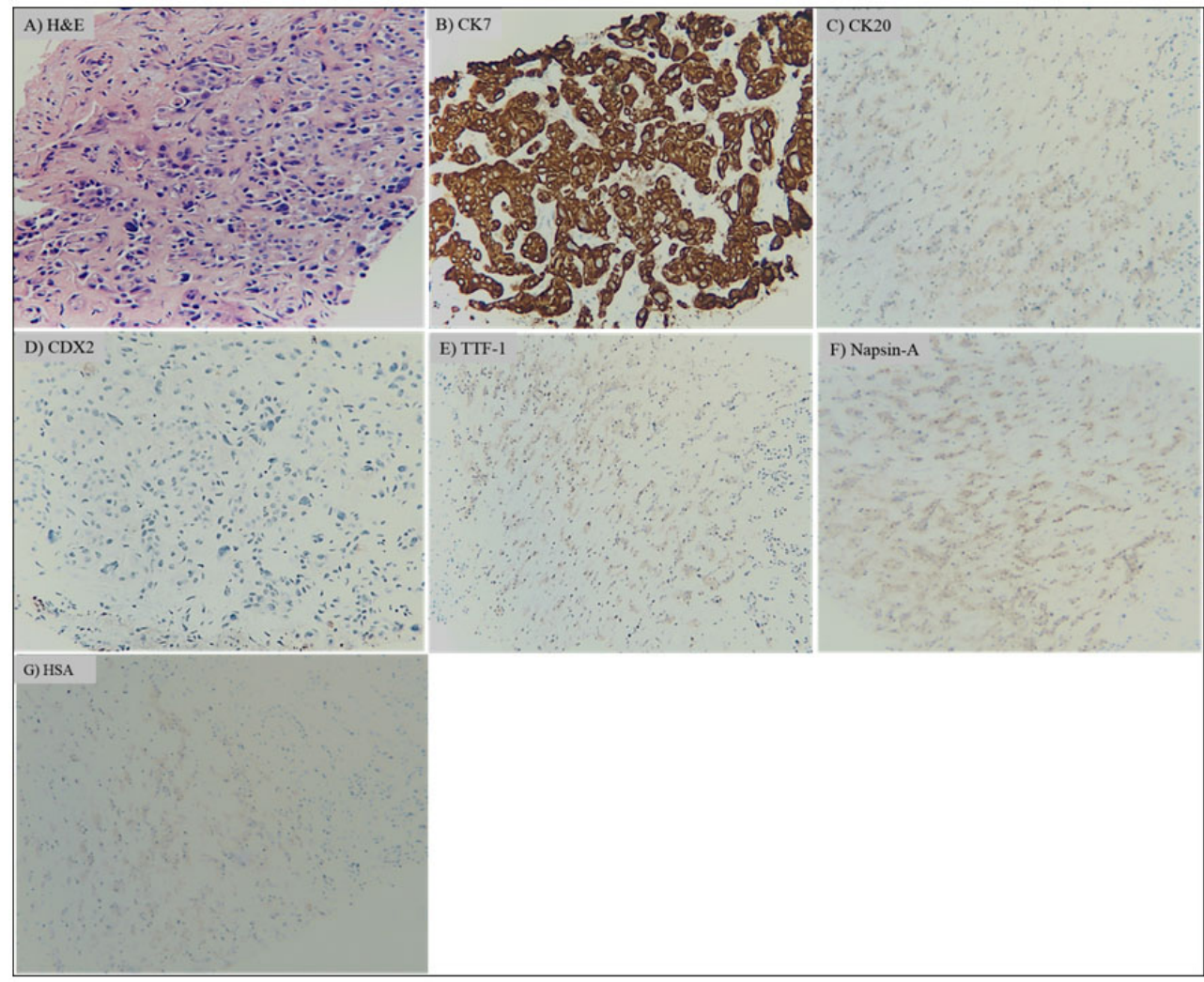

Figure 4. A) H\&E stain 40x showing adenocarcinoma of the liver. B) through G) The immunohistochemical profile: CK7 strongly and diffusely positive (cytoplasmic), CK20 negative, CDX2 negative, TTF-1 negative, napsin A negative, and HAS negative.

\section{DisCUSSION}

We present a unique case of a patient with a KRAS-mutant rectal mucinous adenocarcinoma who subsequently developed two lung adenocarcinomas. One of the lung adenocarcinomas was also KRAS-mutated and showed mucinous morphology whereas the second was KRAS-wildtype and showed acinar-predominant morphology. Afterwards, the patient developed KRAS-mutated hepatic adenocarcioma before dying of acute liver failure. We initially suspected that the mucinous adenocarcinoma found in the lung was a metastasis from the rectal primary based on the shared mucinous morphology. However, the KRAS-mutant mucinous adenocarcinoma in the lung (AGT) had a different genotypic mutation from that of the rectal mucinous adenocarcinoma (GAT), and it was favored that the mucinous adenocarcinoma of the rectum and lung were unrelated.

Certain genetic mutations are correlated with colorectal cancers such as KRAS, BRAF, p53, APC and PIK3CA, among which mutational activation of KRAS codons 12 and 13 is particularly common. ${ }^{[1]}$ KRAS mutations, which occur in approximately $40 \%$ of colorectal adenocarcinomas, correlate with response to therapy, clinical outcome and the pattern of metastatic spread. In this regard, KRAS-mutated colorectal cancers are resistant to targeted anti-epidermal growth factor receptor (EGFR) therapies cetuximab and panitumumab. ${ }^{[3]}$ The clinical course is also shaped by the KRAS mutational 
status. For example, KRAS-mutated colorectal tumors that metastasize to the liver are associated with higher recurrence, decreased recurrence-free survival and overall survival after resection of hepatic metastasis. ${ }^{[4,5]}$ In addition, the presence of KRAS mutation seems to influence site-specific metastasis, whereby KRAS-mutated colorectal tumors tend to metastasize more to the lung and brain and less commonly to the liver. ${ }^{[6]}$ KRAS mutations also occur in $30 \%$ of lung adenocarcinomas. However, contrary to other common mutations such as EGFR and ALK, which occur in $14 \%$ and $7 \%$ of lung adenocarcinomas respectively, there is no therapy targeting KRAS mutations. ${ }^{[2]}$ Compared to EGFR-mutated lung cancers, KRAS mutations correlate with lower frequency of brain metastasis, lower recurrence after surgical resection and yet shorter survival. ${ }^{[8]}$ Overall, KRAS mutations in nonsmall cell lung cancers are associated with poor prognosis. ${ }^{[7]}$

The different mutated KRAS sequences in the rectal and lung mucinous adenocarcinomas raises the question as to whether a metastatic tumor can display a different genotype from that of the primary. In this regard, studies show that while the majority ( $>90 \%$ ) of primary colorectal cancers and their metastatic derivatives are mutationally concordant i.e. share the same mutation status, a minority of cases were discordant which may have contributed to the discrepancy of the treatment response between the primary and metastatic lesions. ${ }^{[9,10]}$ For example, in a study of 43 cases of colorectal tumors matched to their liver metastases, 9 cases had KRAS mutation both in primary and metastatic tumors, 3 cases showed KRAS mutation in the primary colorectal tumors only, and 5 showed KRAS mutation in the liver metastasis only. ${ }^{[11]}$

The afore-mentioned mutational discordance between the primary and metastatic lesions may be reasonably explained by the premise of tumor heterogeneity whereby the tumor consists of several coexistent intra-tumor clones, each with its distinct mutational profile. ${ }^{[12]}$ It is possible that the colonic KRAS mutation reflects that of the preponderant clone in the sampled specimen whereas the cells analyzed in the KRASmutated lung adenocarcinoma belong to a different clone with a different mutation. Another explanation is the genetic instability of tumor cells leading to continual mutational progression with the consequent considerable phenotypic and genotypic evolution. ${ }^{[13]}$

In conclusion, while the tumor heterogeneity hypothesis can explain the discordant KRAS mutation in the lung mucinous adenocarcinoma compared to the previous rectal mucinous adenocarcinoma and hence support a diagnosis of metastasis, the low frequency of intratumoral heterogeneity, the convincing CK20 negativity, and overall lepidic pattern seen in the mucinous adenocarcinoma found in the lung, led us to favor a lung primary over a metastasis from the rectal mucinous adenocarcinoma. In rare circumstances of two tumors showing the same morphology, sometimes checking for gene mutations, and even specific mutation sequence can help differentiate between two different primaries versus a metastasis. Making such a distinction is important for prognosis and treatment approach in these rare circumstances.

\section{REFERENCES}

[1] Janakiraman M, et al. Genomic and biological characterization of exon 4 KRAS mutations in human cancer. Cancer Res. 2010; 70(14): 5901-11. PMid:20570890. https://doi.org/10.1158/0008-5 472. CAN-10-0192

[2] Kerr EM, Martins CP. Metabolic rewiring in mutant Kras lung cancer. FEBS J. 2017.

[3] Amado RG, et al. Wild-type KRAS is required for panitumumab efficacy in patients with metastatic colorectal cancer. J Clin Oncol. 2008; 26(10): 1626-34. PMid:18316791. https://doi.org/10.1 200/JC0.2007.14.7116

[4] Vauthey JN, et al. RAS mutation status predicts survival and patterns of recurrence in patients undergoing hepatectomy for colorectal liver metastases. Ann Surg. 2013; 258(4): 619-26; discussion 626-7.

[5] Karagkounis G, et al. Incidence and prognostic impact of KRAS and BRAF mutation in patients undergoing liver surgery for colorectal metastases. Cancer. 2013; 119(23): 4137-44. PMid:24104864. https://doi.org/10.1002/cncr. 28347

[6] Tie J, et al. KRAS mutation is associated with lung metastasis in patients with curatively resected colorectal cancer. Clin Cancer Res. 2011; 17(5): 1122-30. PMid:21239505. https://doi.org/10.1 158/1078-0432.CCR-10-1720

[7] Riely GJ, Marks J, Pao W. KRAS mutations in non-small cell lung cancer. Proc Am Thorac Soc. 2009; 6(2): 201-5. PMid:19349489. https://doi.org/10.1513/pats.200809-107LC

[8] Tomasini P, et al. EGFR and KRAS Mutations Predict the Incidence and Outcome of Brain Metastases in Non-Small Cell Lung Cancer. Int J Mol Sci. 2016; 17(12).

[9] Vakiani E, et al. Comparative genomic analysis of primary versus metastatic colorectal carcinomas. J Clin Oncol. 2012; 30(24): 295662. PMid:22665543. https://doi .org/10.1200/JC0.2011.38. 2994

[10] Lee SY, et al. Comparative genomic analysis of primary and synchronous metastatic colorectal cancers. PLoS One. 2014; 9(3): e90459.

[11] Kawamata H, et al. Discrepancies between the K-ras mutational status of primary colorectal cancers and corresponding liver metastases are found in codon 13. Genomics. 2015; 106(2): 71-5. PMid:26026309. https://doi.org/10.1016/j.ygeno.2015.05.007

[12] Marusyk A, Polyak K. Tumor heterogeneity: causes and consequences. Biochim Biophys Acta. 2010; 1805(1): 105-17. PMid:19931353.

[13] Leroi AM, Koufopanou V, Burt A. Cancer Selection. Nat Rev Cancer. 2003; 3(3): 226-31. PMid:12612657. https://doi .org/10.103 $8 / \mathrm{nrc1016}$ 